\title{
PROCAMPO \\ Procampo
}

\author{
Cristal Arias Rojas \\ Crariasr@poligran.edu.co \\ David Arenas Remolina \\ Carenas1@poligran.edu.co \\ Juan Sebastián Martínez \\ jsmartinez@poligran.edu.co \\ Profesor
}

Institución Universitaria Politécnico Grancolombiano

Semillero de Formación por Retos

Negocios Internacionales y Comunicación Social y Periodismo

Bogotá, Colombia

\section{RESUMEN}

Procampo tiene el objetivo de convertirse en una plataforma que brinde beneficios a la comunidad campesina del departamento de Cundinamarca en diversas variables críticas para su calidad de vida, creando de esta manera un Hub Tecnológico-Social. Para lograrlo se conformó un equipo de once personas multidisciplinares pertenecientes al grupo de formación por retos de la Institución Politécnico Grancolombiano. Se inició con la investigación de la población en cuanto a sus más grandes características, datos demográficos, cultivos, economía, rutas de mercado, educación, violencia, entre otros, con el objeto de recrear una caracterización de la población rural; luego se seleccionaron tres grandes variables críticas dentro de la población (Producción Agrícola, Educación y Violencia), los cuales se integraron por medio de una plataforma tecnológica de analítica para conformar lo que se denominó un centro de procesamiento de datos rural. Por otro lado, se realizó una investigación y análisis formal de un conjunto de posibles empresas con las que se podría trabajar entorno a sus políticas de responsabilidad social para el desarrollo de proyectos en conjunto, todos estos en pro del logro de cuatro objetivos de desarrollo sostenible (ODS): Fin de la pobreza, Educación de calidad, Trabajo decente y Crecimiento económico y Reducción de desigualdades, teniendo en cuenta que son los ODS en los que busca enfocarse PROCAMPO, sin embargo, no sin posiblemente amparar los demás ODS.

Actualmente, el equipo se encuentra en fase de planeación en cuanto al diseño del backendy frontend para la página Web objetivo; se estima que, a lo largo del primer semestre del año 2021, esté consolidada una plataforma con suficiente interacción para los cultivadores y los compradores. Igualmente, para este mismo semestre se espera contar con una guía metodológica de desarrollo de proyectos sociales que contemple la manera de desarrollar los proyectos de responsabilidad social en las comunidades campesinas.

Palabras clave:

Responsabilidad Social Empresarial, hub, objetivos de desarrollo sostenible, comunidad rural 


\section{INTRODUCCIÓN}

PROCAMPO nace de la necesidad que requiere la población rural de Colombia de satisfacer de manera adecuada sus necesidades básicas y de disminuir la brecha económica y social que se tiene entre las poblaciones rurales y urbanas del país; así mismo, con el objeto de aportar al cumplimiento de los objetivos de desarrollo sostenibles establecidos por la ONU en el país. Para ello, PROCAMPO se cataloga como un hub tecnológico-social que permita ser solidario con la población rural necesitada, y así se crea en conjunto con un semillero de investigación del Politécnico Grancolombiano un proyecto en pro del agro colombiano que busque identificar diversas variables productivas, sociales y económicas para la elaboración de proyectos con sentido social, permitiendo a la población educarse, capacitarse, recibir ayudas físicas o monetarias que les permitan mejorar su calidad de vida; por lo tanto, PROCAMPO busca centralizar los esfuerzos de las empresas que cumplen sus obligaciones de responsabilidad social empresarial, RSE, para el desarrollo de proyectos que beneficien a la población rural de Cundinamarca.

PROCAMPO se encarga de estudiar y caracterizar la población rural de Cundinamarca para generar proyectos en torno al cumplimiento de los ODS (Objetivos de Desarrollo Sostenibles), que permitan mejorar la calidad de vida de la población rural de Cundinamarca, reuniendo empresas que buscan mejorar sus estrategias RSE para generar proyectos en red de empresas con mayor impacto.

Todo esto es construido por estudiantes de diferentes disciplinas como mercadeo y publicidad, psicología, negocios internacionales, ingeniería de sistemas, ingeniería industrial, desarrollo de software, diseño gráfico y comunicación social y periodismo, pasando por etapas de trabajo que se han consensuado entre los integrantes del grupo y el profesor a cargo del semillero, etapas como investigación y caracterización de la población, sistematización de los datos, investigación de empresas potenciales, construcción del modelo de negocio, mockup de diseño para la página, estrategia de abordaje, plan de mercadeo, campaña 360 de publicidad y construcción de la página Web.

\section{MÉTODO}

El desarrollo de este proyecto de investigación es mixto, trabaja elementos de tipo cualitativo y cuantitativo, por lo que se requiere un análisis profundo del entorno y de la población, así como el uso de herramientas cuantitativas para el análisis de datos y variables cuantitativas de impacto en la población.

La información fue obtenida en primera instancia bajo fuentes secundarias, tal como artículos científicos, artículos de revistas informativas, páginas gubernamentales, entre otros. Por otro lado, también se obtuvo información de primera mano por diversas herramientas de opinión y herramientas de percepción a diversos actores. Para la construcción del proyecto se utilizan diferentes matrices para visualizar macroentorno del proyecto, como matrices PESTEL, MEFI, MEFE y DOFA. Por otra parte, utilizamos tres objetivos específicos que están ligados a diferentes entregas.

- Investigación sobre cultivos, características, actores, necesidades y rutas de distribución del agro en el departamento de Cundinamarca.

- Investigar las políticas y requisitos de la responsabilidad social en las empresas colombianas.

- Investigación de posibles prospectos de empresa para el hub.

- Diseño de manual de identidad de marca.

- Construcción de plan de negocios.

- Determinar los variables y requerimientos para la generación de software necesarios para una plataforma tecnológica.

En la llustración 1 se observa el método de trabajo del proyecto de PROCAMPO, teniendo en cuenta tres fases: la primera, un proceso de caracterización de la población y desarrollo de un centro de procesamiento de datos para la elaboración de proyectos; la fase número dos tiene como objetivo el proceso de mercadeo y 
estrategia para la accesibilidad a la población rural y a los sponsors o empresas con RSE. La última relata cómo por medio de la accesibilidad de datos y formulación de proyectos y el análisis de mercadeo se pueden generar los proyectos y desarrollar un proceso de seguimiento, evaluación y cierre de los mismos.

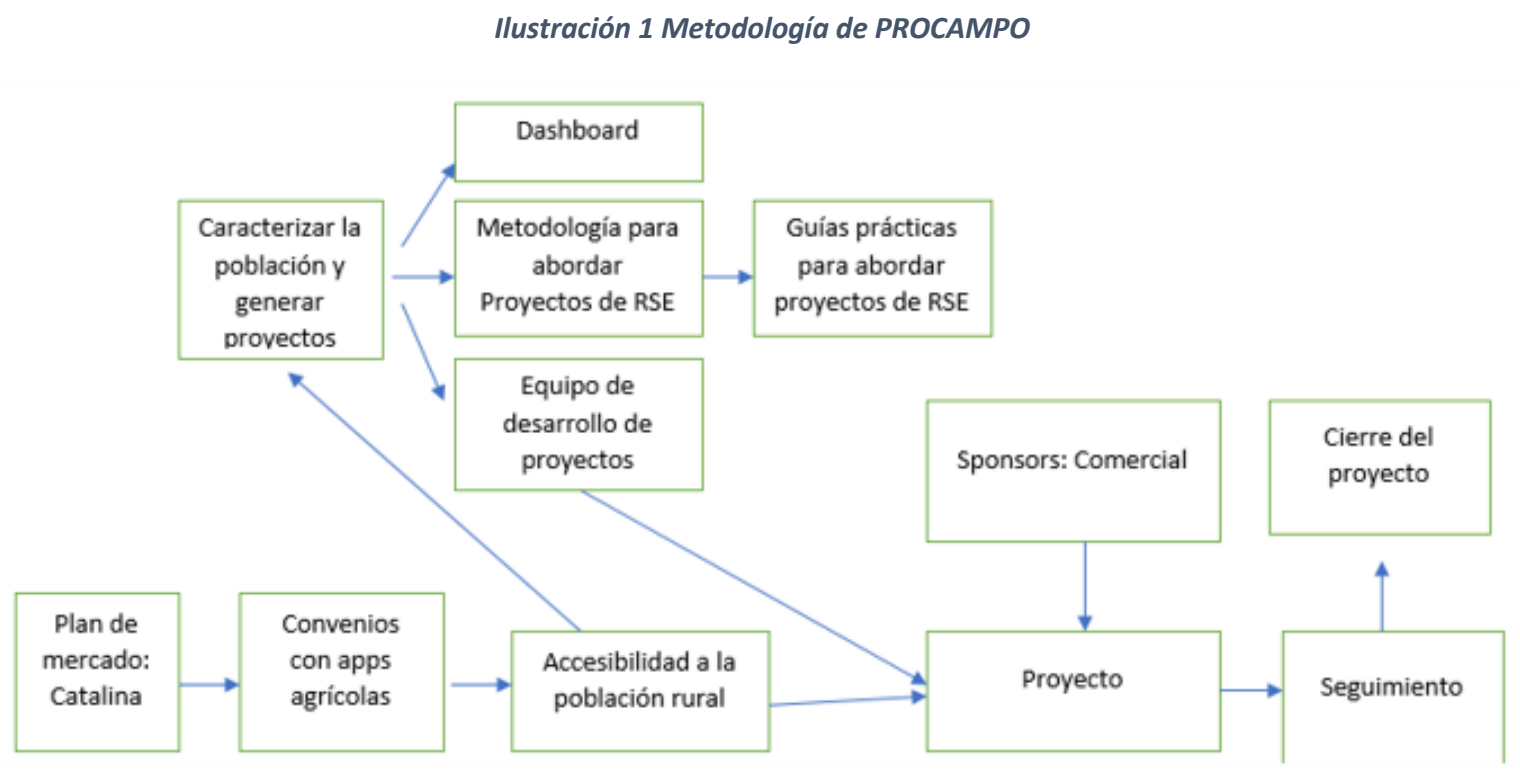

\section{RESULTADOS}

El proyecto aún se encuentra en marcha con una evolución del 40\%, sin embargo, se ha tenido un gran avance en el área investigativa, iniciando con un documento formalizado, en el cual se dejó claro las necesidades y características de cada provincia del departamento de Cundinamarca, que está dividido en 15 provincias que en total suman 116 municipios en la zona; se resaltó las condiciones de educación, esto porque se evidenció que en la etapa de secundaria y educación profesional el porcentaje de personas que siguieron con su educación disminuyó drásticamente. En este análisis se han obtenido importantes resultados en la creación de un centro de procesamiento de datos, reconociendo tres elementos de estudio importantes:

- Producción agrícola: análisis detallado de la producción agrícola en cada una de las provincias de Cundinamarca, reconociendo en el mapa los principales puntos de producción, los hub de producción de cada uno de los cultivos y los productos fuertes en cada una de las zonas de la región de Cundinamarca.

- Educación: se tiene una base de datos detallada de cada uno de los estudiantes que han presentado las pruebas Saber en Cundinamarca en los últimos años, reconociendo las tendencias, así como su caracterización social y demográfica.

- Violencia: se tiene cada uno de los delitos cometidos en la última década en Cundinamarca, reconociendo tendencias, zonas de concentración de cada uno de los delitos y población vulnerada.

Como complemento a este, se pudo especificar la ubicación clara en el mapa de cada provincia junto con su capital y su economía, que en la mayoría se resalta el sector agropecuario, la industria y la ganadería.

El sub-equipo encargado del área de negocios, finanzas y estadísticas pudo definir cuáles son las normativas que, según las leyes del Estado colombiano, se deben conocer para llevar de forma adecuada un proceso de RSE. En esta investigación se pudo evidenciar que no hay muchas normas y leyes que 
regulen la RSE en las empresas, debido a que esta es fruto de iniciativas voluntarias. Se identifica que la misión de la RSE es contribuir al respeto de los derechos humanos de los trabajadores en todo el mundo, mediante la promoción de condiciones de trabajo ético, los derechos laborales, la responsabilidad social corporativa (RSC) y el diálogo social; ISO 2600 habla sobre la percepción que se tenga acerca del desempeño de una organización en materia de responsabilidad social, cómo su desempeño real pueden influir, entre: su ventaja competitiva, reputación, capacidad de mantener a todos sus stakeholders participando para cumplir con estos objetivos sociales; se tuvo en cuenta la Ley 70 de 2010 del Senado. A parte de esto encontramos algunos beneficios para las empresas que trabajan la RSE como aplicar al ranking Merco y beneficios tributarios.

En paralelo con la anterior investigación comentada, se identificaron posibles empresas que cumplen con el perfil para trabajar la RSE junto a PROCAMPO, dando como resultado la identificación de los ODS que más se adecuan a la generación de oportunidad y desarrollo de la población rural de Cundinamarca, dejando así un total de cuatro objetivos específicos a trabajar.

Más adelante, por medio de indagación y análisis de algunos proyectos similares, se reconoció que era importante construir un documento sobre las centrales de abastos que se encuentran en el departamento para contribuir más al factor de la producción agrícola, esto debido a que es por allí por donde ingresan todos los productos que se traen del campo; de igual forma se establecen precios, flujo de productos y el comportamiento de la demanda y la oferta; se pudo detectar que aproximadamente un $55 \%$ de las frutas que llegan a la central de abastos de Bogotá son cosechadas en el área de Cundinamarca; adicionalmente, el $67 \%$ de las hortalizas también son cosechadas en esta región, dejando en claro que gran parte del cultivo campesino se dispone para la capital del país y sus más de siete millones de personas que la habitan.

La construcción de la matriz PESTEL sirvió para encontrar el factor legal, tecnológico, económico y social dentro de Procampo en el ámbito en el que se mueve la misma, con contratos y modificaciones, partes legales y tecnológicas que se dan desde la conectividad a la falta de herramientas tecnológicas en ciertos lugares. La matriz MEFE nos ayudó a obtener -partiendo de lo que estudiamos en el PESTEL- cuáles son las oportunidades con factor de éxito; entonces, en la creación del hub tecnológico dejó saber que es una fortaleza porque La MEFI ayuda a saber el éxito interno que se puede dar en PROCAMPO. Y por último, la matriz DOFA con la que se identificó las debilidades, amenazas, oportunidades y fortalezas que podemos cruzar con la información y hacer unas estrategias para fortalecer el proyecto.

Por último, se reconoce una plataforma Web que se encuentra al 60\% de su desarrollo, reconociendo la misma como funcional para la muestra de los proyectos que pretende desarrollar PROCAMPO, visualiza el centro de procesamiento de datos y permite la generación de usuarios para pertenecientes a la entidad y los sponsors, de manera que se logre un seguimiento a los proyectos en marcha.

\section{DISCUSIÓN Y CONCLUSIÓN}

El cumplimiento y desarrollo de objetivos con responsabilidad social en empresas no es simplemente para generar indicadores macroeconómicos; se debe recalcar la visión de construir una empresa con conciencia y autoanálisis de lo que se puede llegar hacer en la comunidad, reconociendo que genera un impacto y que este debe ser retribuido.

El hub tecnológico social debe ser un movilizador de conciencia en la realidad, medido con impacto real, desarrollo y oportunidad, por lo tanto, PROCAMPO debe fortalecer sus mecanismos de evaluación y seguimiento a sus futuros proyectos sociales, reconociendo y evaluando de manera concienzuda el impacto que brinda a la sociedad.

\section{ENCUENTRO CON SEMILLEROS, APORTES Y REFLEXIONES}


No existe una ley exacta en Colombia que demande a la empresa como obligación a implementar la responsabilidad social, se reconoce una voluntad del gobierno para que las organizaciones se integren a estas prácticas con la retribución en términos tributarios y demás, sin embargo, aún falta un mayor impulso, si bien no obligatorio, sí en torno a la promoción de la RSE.

El hub tecnológico social que se encuentra en desarrollo se tomará varias de las aplicaciones que ya existen y trabajan junto con los agricultores campesinos, con el objetivo de crear una red de empresarios con la finalidad de brindar beneficio al sector que más lo necesita. Para ello, PROCAMPO será el mediador de todo este proceso, creando proyectos de impacto en las comunidades que se encuentren ligadas y con las que se logre convenio; PROCAMPO buscará posicionarse como una de las grandes fundaciones del país, que logre generar alto impacto a la comunidad y de solución y satisfacción a las necesidades básicas de la población rural colombiana.

Se puede afirmar que, para principios del próximo año ya existirá una fase de la página Procampo, y se ha elaborado un plan para seguir trabajando desde allí.

\section{REFERENCIAS BIBLIOGRÁFICAS}

Cavala. (2020). Responsabilidad social empresarial.

Global competitiveness report. (2020). Global competitiveness report Colombia taxes coporatives.

Gobierno Nacional de Colombia. (2006). Proyecto de Ley número 153 de 2006 senado, 153 .

La República. (2018). Normas que regulan los planes de sostenibilidad o de responsabilidad social en Colombia .

Ley Colombia. (2020). Información Proyecto de ley 70 de 2010 Senado.

Mojica-Montoya, LF. (2018). Factores de equidad en las unidades agrícolas familiares del Municipio de la Calera. Colombia: Editorial Fundación Universitaria San Mateo.

Mercosur (2019). Monitor empresarial de reputación corporativa.

Organización de las Naciones Unidas. (s.f.). Pacto Global y los Objetivos de Desarrollo Sostenible ODS. 2020 . 